\title{
Atrofia de íris após tratamento estético facial com luz intensa pulsada
}

\section{Iris atrophy after aesthetic treatment with intense pulsed light}

Ângelo Ferreira Passos ${ }^{1}$, Daniele Fioroti Borges ${ }^{2}$

\section{RESUMO}

Relato de um caso de complicação ocular, em consequência do uso de luz intensa pulsada, para tratamento facial cosmético. A lesão consistiu em atrofia iriana no setor temporal, com grande área de transiluminação, sinéquias posteriores, deformidade e redução da dilatação pupilar. O objetivo é alertar para os riscos do procedimento para os olhos, caso não sejam tomadas as medidas adequadas de proteção ocular.

Descritores: Terapia a laser/efeitos adversos; Iris/lesões; Irite; Transiluminação; Atrofia; Relatos de casos

\begin{abstract}
The authors report a case of ocular complication, following Intense Pulsed Light for cosmetic facial treatment. The lesion was iris atrophy with a large transilumination area, posterior synechiae, deformity and reduced dilation of the pupil, on the temporal side. The aim is to alert for the risk of ocular lesion related to this procedure, if adequate measures to protect the eyes are not taken.
\end{abstract}

Keywords: Laser therapy/adverse effects; Iris/injuries; Irite; Transilumination; Atrophy; Case reports

\footnotetext{
${ }^{1}$ Universidade Federal do Espírito Santo - UFES - Vitória (ES), Brasil; Hospital Universitário Cassiano Antônio de Moraes - Vitória (ES), Brasil;

${ }^{2}$ Hospital Universitário Cassiano Antônio de Moraes - Vitória (ES), Brasil.

Hospital Universitário Cassiano Antônio de Moraes da Universidade Federal do Espírito Santo - UFES - Vitória (ES), Brasil.
}

Os autores declaram inexistir conflitos de interesse

Recebido para publicação em 19/12/2010 - Aceito para publicação em 28/8/2011 


\section{INTRODUÇÃO}

A procura por tratamentos para rejuvenescimento vem crescendo, aumentando o número de procedimentos a laser, já que este é uma boa opção terapêutica, por ser pouco invasivo, de rápida execução e recuperação além de ter bons resultados. ${ }^{(1)}$

O Laser já tem seus benefícios definidos no tratamento de vários problemas, como despigmentação, alteração da textura da pele, rugas, telangiectasias e remoção de pelos. ${ }^{(1,2)}$ Outra fonte de luz comumente utilizada e com os mesmos benefícios é a Luz Intensa Pulsada (Intense Pulsed Light - IPL), que utiliza um feixe de luz não coerente, com comprimento de onda que varia de $500-1200 \mathrm{~nm}$, dependendo do tratamento a ser realizado. ${ }^{(3)}$

Com o aumento do número de procedimentos utilizando IPL, observou-se também a elevação do número de efeitos adversos. Na maioria das vezes, esses são transitórios, como dor, eritema, vesículas ou crostas superficiais, nos sítios de tratamento, podendo haver também hipo ou hiperpigmentação. ${ }^{(4)}$

O objetivo desse artigo é relatar a ocorrência de um caso de lesão ocular definitiva relacionado ao uso do $I P L$, alertando para a necessidade de medidas adequadas de proteção. Foram encontrados apenas dois relatos na literatura de lesão semelhante, causada por este tipo de tratamento.

\section{Relato do caso}

Paciente do sexo feminino, branca, de 45 anos, atendida em consulta de rotina, em abril de 2010, com histórico de que, há oito meses, havia sido submetida a tratamento estético dermatológico facial, inclusive na região em torno dos olhos com laser. Relatou que, algumas horas após o tratamento, apresentou quadro de forte dor ocular e turvação visual, sendo que esses sintomas eram mais proeminentes no olho esquerdo. Na ocasião, foi medicada por especialista, tendo sido prescritos analgésicos e medicação tópica, que não soube precisar. O quadro ocular persistiu por alguns dias, mas não houve retorno ao médico para reavaliação. O tratamento realizado foi $I P L$, com o equipamento Harmony, Alma Lasers Ltd., Israel. Utilizou-se o comprimento de onda de $690 \mathrm{~nm}$, sem uso de proteção ocular.

Durante consulta de rotina, informou acuidade visual de 20/20 em ambos olhos, com correção de +2.25 esférico, e J1 com adição de +1.50 . Ao exame biomicroscópico apresentava, no olho esquerdo, conjuntiva e córnea sem alterações, câmara anterior sem reação, atrofia iriana temporal, mais intensa na porção ciliar que na pupilar, estendendo-se aproximadamente de 1:00 às 3:00 horas. $O$ defeito era caracterizado por intensa transiluminação iriana, denotando acentuada lesão do epitélio pigmentário (Figura 1). Havia sinéquias posteriores e a pupila apresentava discreta irregularidade, com significativa redução da mobilidade e da dilatação na área correspondente à lesão. Por outro lado, a superfície anterior da íris não apresentava lesão aparente, sugerindo que não tinha ocorrido alteração significativa no estroma (Figura 2). Não foram observadas alterações no cristalino.

Fundoscopia em ambos olhos apresentava papila fisiológica, sem alterações na retina do polo posterior e da periferia.

A paciente informava que passou a apresentar fotofobia após ter sido submetida ao tratamento com IPL.

\section{DiscussÃo}

A IPL é uma forma não invasiva de tratamento rejuvenescedor, atuando não só em tecidos pigmentados, como também em lesões vasculares, como as telangiectasias. O método também é utilizado para depilação. $^{(1,2)}$

O equipamento de $I P L$ emite amplo espectro de luz, com comprimentos de onda que variam de 500 a 1200 nanômetros, em pulsos curtos. ${ }^{(5)}$ Diferentes tecidos absorvem diferentes comprimentos de onda. Por isso, filtros são utilizados na tentativa de selecionar o comprimento de onda ideal para o tecido ou lesão a ser tratada. O intervalo entre os pulsos também é ajustado, para manter a área tratada aquecida e diminuir o dano às estruturas vizinhas. ${ }^{(3)}$

A íris possui grande quantidade de pigmento e absorve amplo espectro de comprimentos de onda (faixa de 400-750 nm), o que a torna uma estrutura extremamente vulnerável ao IPL. ${ }^{(3)}$ Assim, se for atingida, pode apresentar inflamação, atrofia, perda de pigmento, transiluminação, sinéquias e distorção pupilar, ${ }^{(1,3)} \mathrm{com}$ alteração da dilatação. A disfunção iriana pode resultar em aumento da fotossensibilidade, lacrimejamento, diminuição da percepção de contraste, dificuldade de adaptação ao claro-escuro e glare. ${ }^{(3)}$ A paciente em questão queixava-se de fotofobia.

O caso do presente relato se refere a uma lesão ocular típica por $I P L$, tendo em vista sua semelhança com lesões comprovadamente causadas pelo mesmo tipo de tratamento $^{(1,3)} \mathrm{O}$ comprimento de onda utilizado foi de $690 \mathrm{~nm}$ e, portanto, dentro do espectro absorvido pela íris. ${ }^{(3)}$

Foram encontrados dois relatos de caso com le- 


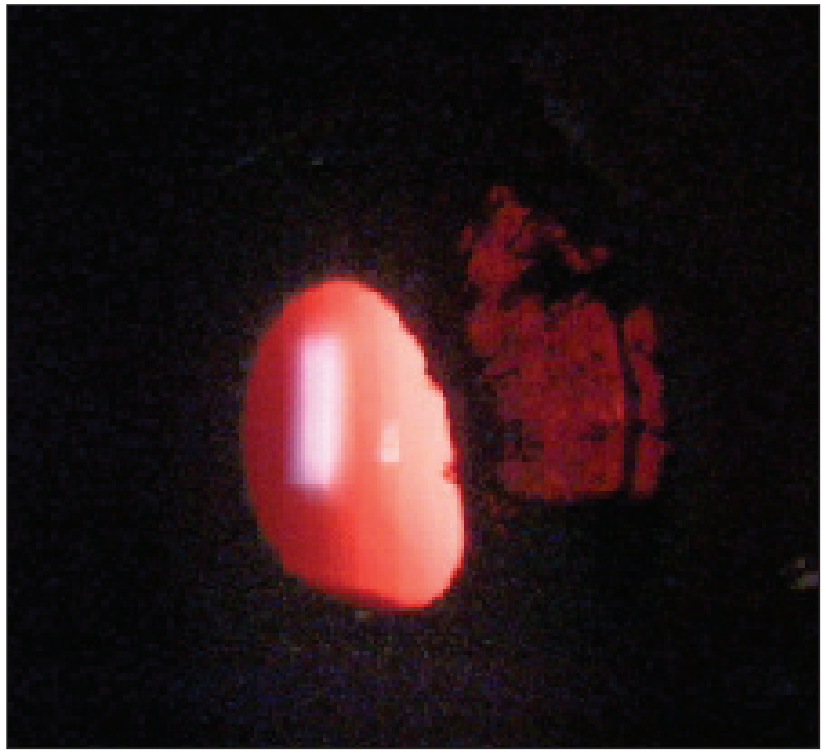

Figura 1: Atrofia no setor temporal da íris do olho esquerdo, com sinéquias, irregularidade da borda e limitação da dilatação pupilar, na área correspondente à atrofia

são iriana em consequência de tratamento dermatológico pelo $I P L$, sendo um deles unilateral ${ }^{(3)} \mathrm{e} \mathrm{o}$ outro bilateral. ${ }^{(1)}$ No presente caso, como nos outros dois encontrados, houve lesão setorial da íris, com deformidade pupilar e redução localizada da dilatação pupilar, por sinéquias e atrofia iriana, mais evidente no epitélio pigmentário, mas podendo também ter ocorrido lesão muscular. Também, como nos outros casos relatados, não foram observadas outras alterações oculares, como catarata e glaucoma, que poderiam ter ocorrido, dependendo do grau de queimadura e inflamação intraocular.

No presente caso, a íris era clara, com forte tonalidade azul, e, portanto, com menos pigmento no estroma, fato que pode ter minimizado a lesão dessa camada. A paciente era acompanhada desde 1992, por um dos autores do presente relato (AFP), e antes da constatação da referida lesão, em abril de 2010, havia sido examinada em abril de 2008. Nesse intervalo, além do episódio relacionado com o citado tratamento, nega qualquer tipo de evento ocular, assim como alterações sistêmicas ou oculares, ou antecedentes de qualquer natureza que pudessem justificar a lesão iriana.

A ocorrência de mais esse caso de complicação ocular pelo uso da $I P L$ confirma a necessidade de que esse tipo de tratamento seja realizado não apenas por pessoal treinado e habilitado ${ }^{(6)}$, mas consciente dos riscos. Para prevenção de complicações oculares, devem ser utilizados óculos opacos, bem ajustados e com proteção lateral quando o tratamento com IPL for realizado

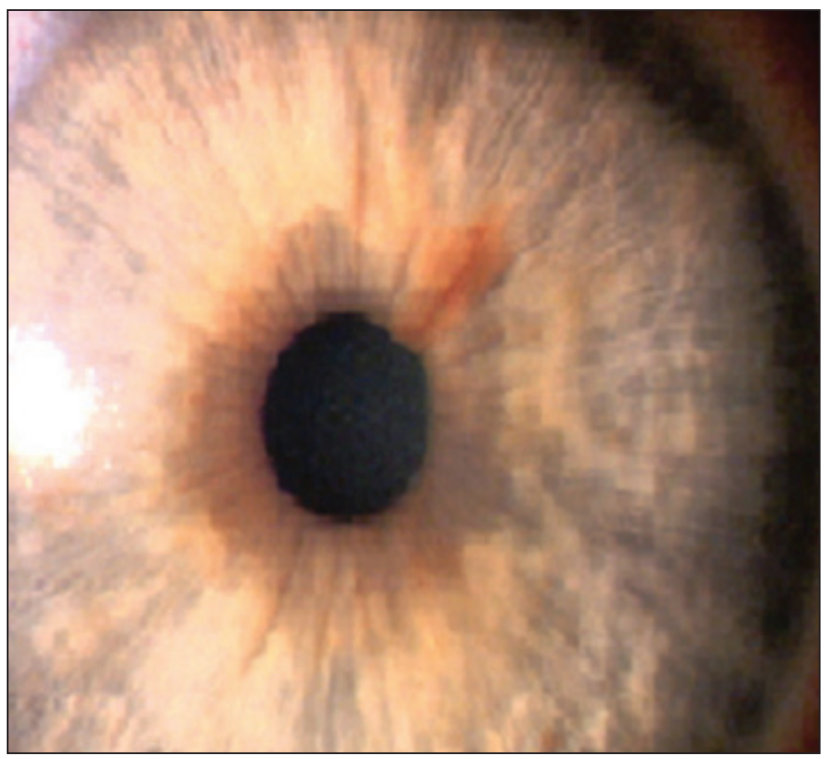

Figura 2: Estroma superficial da íris do olho esquerdo, sem alterações evidentes à biomicroscopia

na região do pescoço, face e couro cabeludo. Quando o procedimento for realizado nas pálpebras, devem ser utilizadas lentes opacas esclerais, para completa proteção do globo ocular. ${ }^{(3)}$ Javey et al. ${ }^{(3)}$ recomendam que esses dispositivos de proteção devem ser feitos de aço inoxidável para que dispersem a energia do IPL.

\section{ReferÊnCIAS}

1. Pang AL, Wells K. Bilateral anterior uveitis after intense pulsed light therapy for pigmented eyelid lesions. Dermatol Surg. 2008;34(9):1276-9.

2. Uebelhoer NS, Dover JS. Photodynamic therapy for cosmetic applications. Dermatol Ther. 2005;18(3):242-52.

3. Javey G, Schwartz SG, Albini TA. Ocular complication of intense pulsed light therapy: iris photoablation. Dermatol Surg. 2010;36(9):1466-8

4. Moreno-Arias GA, Castelo-Branco C, Ferrando J. Side-effects after IPL photodepilation. Dermatol Surg. 2002;28(12):1131-4.

5. Trelles MA, Allones I, Velez M. Non-ablative facial skin photorejuvenation with an intense pulsed light system and adjunctive epidermal care. Lasers Med Sci. 2003;18(2):104-11.

6. Greve B, Raulin C. Professional errors caused by lasers and intense pulsed light technology in dermatology and aesthetic medicine: preventive strategies and case studies. Dermatol Surg. 2002;28(2):156-61.

\section{Endereço para Correspondência:}

Daniele Fioroti Borges

Rua Affonso Claudio, $n^{\circ} 59$ apto 1102 - Praia do Canto

CEP 29055-570 Vitória - (ES), Brasil

E-mail: danielefioroti@hotmail.com 